\title{
An Early-Stopping Mechanism for DSCF Decoding of Polar Codes
}

\author{
Ilshat Sagitov and Pascal Giard \\ LaCIME, Electrical Engineering Department, \\ École de technologie supérieure (ÉTS), Montréal, Canada \\ Email: ilshat.sagitov.1@ens.etsmtl.ca, pascal.giard@etsmtl.ca
}

\begin{abstract}
Polar codes can be decoded with the low-complexity successive-cancellation flip (SCF) algorithm. To improve errorcorrection performance, the dynamic successive-cancellation flip (DSCF) variant was proposed, where the resulting errorcorrection performance is similar to that of the successivecancellation list algorithm with low to moderate list sizes. Regardless of the variant, the SCF algorithm exhibits a variable execution time with a high (worst-case) latency. In this work, we propose an early-stopping metric used to detect codewords that are likely undecodable such that the decoder can be stopped at earlier stages for those codewords. We then propose a modified version of the DSCF algorithm that integrates our early-stopping metric that exploits the specific properties of DSCF. Compared to the original DSCF algorithm, in the region of interest for wireless communications, simulation results show that our proposed modifications can lead to reductions of $22 \%$ to the average execution time and of $45 \%$ to the execution-time variance at the cost of a minor error-correction loss of approximately $0.05 \mathrm{~dB}$.
\end{abstract}

\section{INTRODUCTION}

Polar codes, introduced in [1], are a type of linear errorcorrection codes which can achieve the channel capacity for practically relevant channels. The original successivecancellation (SC) decoding algorithm has low complexity, but its error-correction performance is lacking for many practical applications. To address this, the successive-cancellation list (SCL) decoding algorithm was proposed. It provides great error-correction capability to the extent that polar codes were selected to protect the control channel in 3GPP's nextgeneration mobile-communication standard $(5 \mathrm{G})$, where the SCL algorithm serves as the error-correction performance baseline [2]. The great error-correction performance of SCL comes at the cost of high hardware implementation complexity and low energy efficiency [3].

As an alternative, the successive-cancellation flip (SCF) decoding algorithm was proposed in [4]. The SCF algorithm can be summarized as the application of multiple SC decoding trials, where each trial, beyond the first, flips the value of one intermediate decision. Which value-or bit-to flip is determined from a list that is meant to keep track of the leastreliable decisions, and the latency of an SCF decoder is a function of the maximum number trials. The SCF decoding algorithm leads to an improved error-correction performance compared to an SC decoder, but still falls short of that of an SCL decoder with a moderate list size. However, compared to an SCL decoder, an SCF decoder is more efficient both in terms of computing resources and energy requirements [5].
The dynamic successive-cancellation flip (DSCF) decoding algorithm was proposed in [6], where the authors introduced two major modifications to the original SCF algorithm with the goal of improving the error-correction performance. Firstly, the DSCF algorithm takes a new, more accurate, approach on the metric computations used to establish the list of the leastreliable decisions. Secondly, that algorithm allows for multiple bit flips per trial. With these modifications, the error-correction performance becomes close to that of SCL decoder with small to moderate list sizes [7].

Regardless of these improvements, both SCF and DSCF decoders have a variable execution time by nature, and the variance on that execution time can be significant. This poses a challenge in the realization of receivers, where fixed-time algorithms are preferred. A high variance on the execution time of the decoder leads to large buffers. Alternatively, the maximum number of trials can be reduced, dynamically or not, at the cost of a reduced error-correction performance.

Contributions: In this work, we present modifications to the original DSCF decoding algorithm to integrate an earlystopping mechanism that attempts to distinguish undecodable codewords from decodable ones based on a new early-stopping metric combined with a pre-calculated threshold. Based on that metric, a codeword may be classified as likely undecodable, in which case the decoder attempts a limited number of trials, much lower than the maximum number of trials. After those trials, if decoding is not successful, the decoder stops. The behavior of the decoder for the other codewords remains unaffected. As a result of our modifications to the DSCF algorithm, the average execution time and its variance are reduced at a cost of a minor error-correction performance loss.

Outline: The remainder of this paper is organized as follows. Section $\Pi$ provides a theoretical overview on polar codes, their construction and the SC decoding algorithm. In addition to that, the SCF and DSCF decoding algorithms are briefly presented. In Section III the proposed modifications to the DSCF algorithm are explained, and the new metric along with its calculations used for early stopping is presented. In Section IV the methodology for obtaining the metric distribution over the required number of trials and defining thresholds is explained. In Section $\nabla$ the proposed modified DSCF algorithm is compared against the original one in terms of execution time and error-correction performance. Section VI concludes this work and proposes potential future works. 


\section{BACKGROUND}

\section{A. Construction of Polar Codes}

Polar codes are based on the concept of channel polarization. As the code length tends to infinity, bit locations either become completely reliable or completely unreliable. To construct a $\mathcal{P}(N, k)$ polar code, where $N$ is the code length and $k$ the number of information bits, the $(N-k)$ leastreliable bits are set to predefined values, typically all zeros. These bits are called the frozen bits. The sets of informationand frozen-bit locations are often denoted as $\mathcal{A}$ and $\mathcal{A}^{C}$, respectively. The encoding is the linear transformation such that $\boldsymbol{x}=\boldsymbol{u} \times F^{\otimes n}$, where $\boldsymbol{x}$ is the polar-encoded row vector, $\boldsymbol{u}$ is a row vector of length $N$ that contains the $k$ information bits in their predefined locations as well as the frozen bit values, $n=\log _{2} N$ and $F^{\otimes n}$ is the $n^{t h}$ Kronecker product $(\otimes)$ of the binary polar-code kernel $F=\left[\begin{array}{ll}1 & 0 \\ 1 & 1\end{array}\right]$. The bit-location reliabilities depend on the channel type and conditions. In this work, the additive white Gaussian noise (AWGN) channel is considered and the polar-code construction method used is that of Tal and Vardy [8].

\section{B. Successive-Cancellation Decoding}

Binary trees are a natural representation of polar codes. Fig.1 illustrates the tree-based representation of a $\mathcal{P}(8,4)$ polar code. A polar code of length $N$ can be seen as a composition of two smaller constituent polar codes of length $N / 2$. The color of the leaves indicate whether a location is that of a frozen or information bit. Frozen bits are colored white and information bits are set to black. The SC decoding algorithm follows the depth-first traversing schedule of the tree, visiting the left child nodes first and then passing the calculated messages to the right child nodes. The messages passed to the child nodes are vectors of log-likelihood ratios (LLRs), while those passed to parents are vectors of bits, often called partial-sum bits. These messages are denoted as $\alpha$ and $\beta$, respectively.

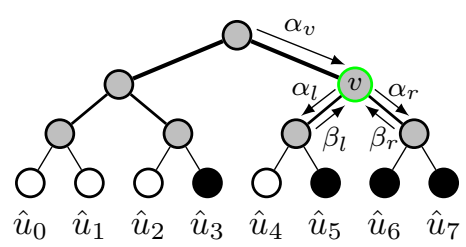

Fig. 1: Tree-based representation of $\mathcal{P}(8,4)$.

By taking the node indicated with $v$ in the decoding tree, the messages to its left $l$ and right $r$ children are calculated according to Eq. (1). The calculations of $f$ and $g$ functions are provided in Eq. (2) for arbitrary real values $x$ and $y$, and binary value $b$.

$$
\begin{aligned}
\alpha_{l}[i] & =f\left(\alpha_{v}[i], \alpha_{v}\left[i+N_{v} / 2\right]\right) \\
\alpha_{r}[i] & =g\left(\alpha_{v}[i], \alpha_{v}\left[i+N_{v} / 2\right], \beta_{l}[i]\right)
\end{aligned}
$$

$$
\begin{aligned}
& f(x, y)=\operatorname{sign}(x) \cdot \operatorname{sign}(y) \cdot \min (|x|,|y|) \\
& g(x, y, b)=(1-2 b) \cdot x+y
\end{aligned}
$$

The value $\beta_{l}$ in Eq. (1) is the estimated bit vector coming to the node $v$ from its left child. In the leaf nodes the estimated bits are calculated with a hard decision on the input LLRs. These LLRs are called the decision LLRs and are denoted as $\alpha_{d e c}$. In the case of frozen bits, hard decisions are not needed, and those bits are directly set to the all-zero vector. For the intermediate nodes, the partial-sum bits are calculated as follows:

$$
\beta_{v}[i]= \begin{cases}\beta_{l}[i] \oplus \beta_{r}[i] & \text { if } i<\frac{N_{v}}{2} \\ \beta_{r}[i] & \text { otherwise }\end{cases}
$$

\section{Successive-Cancellation Flip Decoding}

In [4], at first, the authors designed an Oracle-assisted decoder and showed that the error-correction performance would significantly improve if the first erroneously-estimated bit could be detected and corrected before resuming SC decoding. Then based of these results, they proposed the SCF decoding algorithm. In order to detect the decoding failure of the codeword, the information bits are concatenated with cyclic-redundancy check (CRC) bits of the length $r$ thus increasing the code rate of the polar code to $R=(k+r) / N$. The list of bit-flipping candidates $\mathcal{L}_{\text {flip }}$ is constructed, if its CRC check fails after the first SC decoding pass, based on the least-reliable bit indices, i.e., indices with the smallest absolute $\alpha_{d e c}$ values of the non-frozen bits after the first SC trial. For each new SC trial the next bit index of $\mathcal{L}_{\text {flip }}$ is chosen and when this bit is estimated, the opposite decision is made, i.e., the estimated bit is flipped. Decoding is considered successful if the CRC matches. In order to constrain the latency, a maximum number of trials $T$ is defined, where $T$ is an integer number such that $0 \leq T<(k+r)$. Setting $T$ to 0 renders the $\mathrm{SCF}$ decoder equivalent to an $\mathrm{SC}$ decoder. The list of bit-flipping candidates consists of $\mathcal{L}_{\text {flip }}=\left\{i_{1}, i_{2}, \ldots i_{T}\right\}$, where $i$ is the index of the bit-flipping candidate. The metric associated to each non-frozen bit is defined as:

$$
M_{i}=\left|\alpha_{\text {dec }}[i]\right| .
$$

The resulting metrics are sorted in ascending order and the first $T$ metrics are chosen to form the metric list $M^{T}$. Then the list of the bit-flipping candidates $\mathcal{L}_{\text {flip }}$ associated to the metric list is constructed. The SCF decoder has $T$ additional trials beyond the initial SC pass to decode the codeword while applying the next bit from $\mathcal{L}_{\text {flip }}$ each trial. If decoding is not successful after trial $T$, decoding is stopped and the codeword considered undecodable.

\section{Dynamic Successive-Cancellation Flip Decoding}

The DSCF decoder constructs the list of the bit-flipping candidates differently compared to the SCF algorithm [6]. The metric calculated for each bit is the probability of the event 
that this bit is wrongly estimated and that every previous nonfrozen bits are estimated correctly. In other words, the metric calculated for the current bit indicates the probability that this bit is the first channel-induced error which cannot be corrected by the SC decoding algorithm [6]. The metric can be calculated in both linear or logarithmic domain. Throughout this work, the logarithmic domain computations are considered. The metric calculation for each non-frozen bit after the initial $\mathrm{SC}$ attempt is defined as:

$$
M_{i}=\left|\alpha_{\operatorname{dec}}[i]\right|+\frac{1}{c} \cdot \sum_{\substack{j \leq i \\ j \in \mathcal{A}}} \ln \left(1+e^{\left(-c \cdot\left|\alpha_{\operatorname{dec}}[j]\right|\right)}\right),
$$

where $\ln (\cdot)$ denotes the natural $\log$ arithm, and $c$ is a constant optimized experimentally by way of simulation. The value $c$ will vary depending on the polar code parameters and channel conditions.

In addition to the change in metric computations, the DSCF decoding algorithm allows for multiple bit flips per trial. Instead of a single bit index as an element of the list of bitflipping candidates, now the set of bit indices is considered as one element. One set is denoted with $\epsilon, \epsilon=\left\{i_{1}, i_{2}, \ldots i_{\omega}\right\}$ and $\mathcal{L}_{\text {flip }}=\left\{\epsilon_{1}, \epsilon_{2}, \ldots \epsilon_{T}\right\}$, where $\omega$ is called the order of the decoder, i.e., the maximum number of bit flips within one candidate set. For calculation of metrics associated to sets, we refer the reader to [6] as they are not used in this paper. Indeed, in this work, $\omega=1$ is considered, and the decoding algorithm used could thus be denoted as DSCF-1. However, for the sake of simplicity, in the remainder of this paper, DSCF is also synonymous of the DSCF-1 algorithm unless stated otherwise.

\section{Proposed Algorithmic ModificAtions}

\section{A. Introduction of the Early-Stopping Metric}

The core idea of our proposed method is an early-stopping mechanism that attempts to distinguish the undecodable codewords from the decodable ones. When a codeword that is likely undecodable is identified, our decoder uses a lower maximum number of trials. The early-stopping metric $\phi$ used to identify undecodable codewords is the variance of the elements of $M$ associated to the list of bit-flipping candidates. It is calculated as follows:

$$
\phi=V_{M}=\frac{1}{T-1} \sum_{i=1}^{T}\left(m_{i}-m_{a v}\right)^{2},
$$

where $m_{i}$ is the metric associated to the bit index from $\mathcal{L}[i]$, and $m_{a v}$ is the mean of the elements of $M$ calculated as:

$$
m_{a v}=\frac{1}{T} \sum_{i=1}^{T} m_{i}
$$

The early-stopping metric $\phi$ is not to be confused with metrics associated with the bit-flipping candidates of the DSCF decoder. The metric $\phi$ of our proposed algorithm aims to distinguish the codewords that are likely undecodable from the decodable ones.

\section{B. DSCF Decoder with Early Stopping}

The early-stopping metric $\phi$ explained in previous subsection can be integrated to the DSCF decoder and is calculated for the current codeword after obtaining the list $M$. Then the resulting metric is compared against the pre-calculated threshold metric $\phi_{t h r}$. If $\phi>\phi_{t h r}$, the maximum number of trials becomes the pre-defined reduced maximum number of trials $T=T_{\text {red }}$. Otherwise, the initial maximal number of trials $T$ is kept. The simulation set-up is created and its corresponding algorithm is summarized in Alg.1.

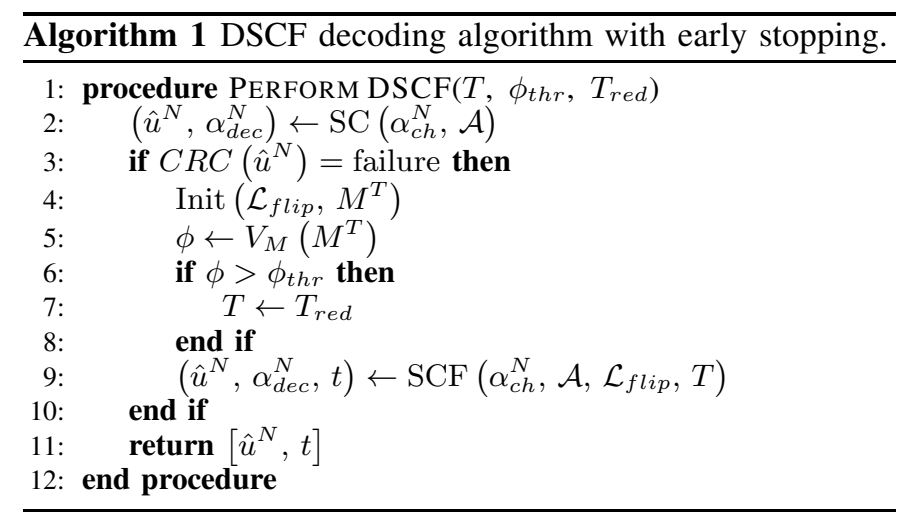

Simulations are for various signal-to-noise ratio (SNR) points. The output number of trials $t$ can be used to build statistics of the execution time averaged over the number of simulation cycles (simulated codewords). The average execution time $T_{a v}$, in terms of average of number of trials, can be calculated as:

$$
T_{a v}=\frac{1}{S} \sum_{i=1}^{S} t_{i}
$$

where $S$ corresponds to the number of simulated codewords per SNR point.

The variance of the execution time $V_{T}$ can also be obtained from the output $t$ and is calculated as follows:

$$
V_{T}=\frac{1}{S-1} \sum_{i=1}^{S}\left(t_{i}-T_{a v}\right)^{2} .
$$

Simulation results are provided in Section $\mathrm{V}$

\section{Obtaining the Metric Threshold From the EARLY-STOPPING METRIC Distribution}

The DSCF algorithm with an early stopping requires the threshold metric $\phi_{t h r}$ and the reduced maximum number of trials $T_{r e d}$, values determined by way of simulation. At first, the distribution of the average $\phi$ is obtained as a function of the number of trials $t$ required by the original DSCF decoder. Then the corresponding thresholds can be defined based on the resulting distribution.

\section{A. Methodology}

The simulation setup created for obtaining the distribution of the average metric depending on $t$ uses random codewords encoded by a $\mathcal{P}(1024,512)$ polar code and a CRC of $r=16$ 


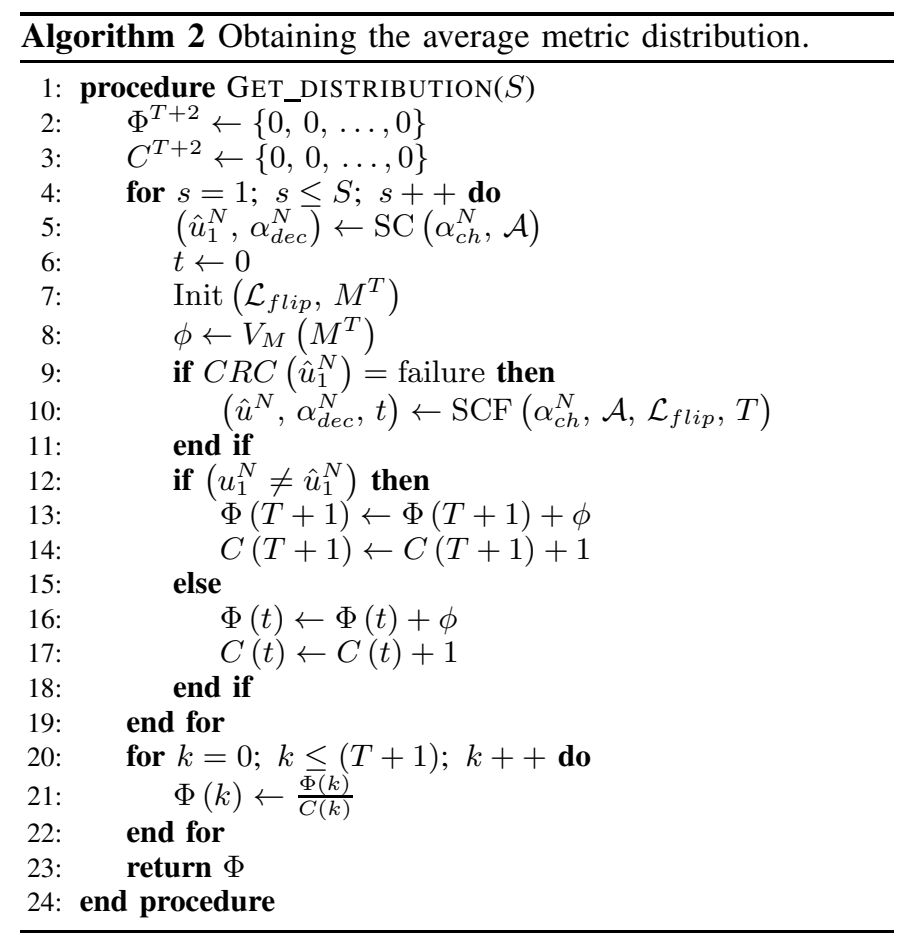

bits with polynomial $z^{16}+z^{15}+z^{2}+1$. Those polar-code length and rate, and that CRC length were chosen to ease comparison with other works as they are commonly found in the literature. The polar code is constructed for an approximate design SNR $\gamma_{\text {des }}$ of $2.365 \mathrm{~dB}$. Binary phase-shift keying modulation is used over an AWGN channel. For each SNR point, the simulation is run for $10^{7}$ codewords. The maximum number of trials beyond the initial SC decoding pass of the DSCF decoder is $T=$ 10. The value $c$ used in the calculation of the DSCF metrics associated to the bit-flipping candidates of Eq. (5) is 0.3 .

\section{B. Average Early-Stopping Metric Distribution}

Alg.2 shows the algorithm used to obtain the average earlystopping metric distribution, where $s$ stands for the simulation index and $S$ corresponds to the total number of simulated codewords. The list of metrics $\Phi$ has length $T+2$ and includes the resulting early-stopping metrics obtained from the successful initial SC trials $t=0$, those corresponding to undecodable codewords, and those for each $1 \leq t \leq T$ corresponding to successfully-decoded codewords. It can be seen that the metric $\phi$ is calculated on line 8 and is accumulated into the list $\Phi$ at the appropriate position based on $t$, either on line 13 or 16 if decoding is successful or not, respectively. The list of counters $C$, which has the same length as $\Phi$, increments its element according to the output $t$ on line 17 in the case of successful decoding or on line 14 otherwise. At the end of the simulation, the elements of the list of accumulated metrics $\Phi$ are normalized by their corresponding counters to obtain the average value. This simulation is ran for each SNR point.

The bar diagram of the resulting average early-stopping metric distribution is presented in Fig.2 for $\gamma=2.25 \mathrm{~dB}$. From this figure, it can be seen that the average early- stopping metrics are distributed exponentially within the range $1 \leq t \leq 10$. The average early-stopping metric corresponding to undecodable codewords is clearly higher than those of decodable codewords with the highest values of $t$ and is close to that of $t=2$.

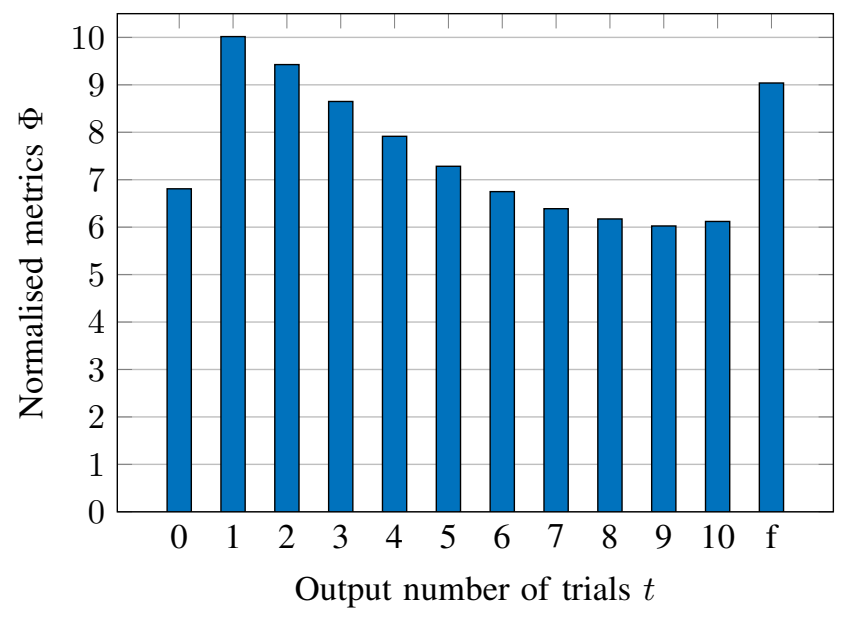

Fig. 2: Distribution of early-stopping metrics depending on the number of trials required by the original DSCF decoder for a $\mathcal{P}(1024,512)$ polar code, a CRC of $r=16$ bits, and a AWGN channel with SNR $\gamma=2.25 \mathrm{~dB}$.

\section{Defining the Threshold Metrics and the Reduced Number of Maximum Trials}

According to the distribution of the average early-stopping metrics shown in Fig. 2] the threshold metric $\phi_{t h r}$ gets the value of the average metric obtained for the undecodable codewords (denoted as " $f$ " in figure). A threshold metric $\phi_{t h r}$ is defined for each SNR point of interest. The relative position of the normalized metric for the undecodable codewords has been observed to be independent from the channel SNR. As a consequence, a single value for the reduced maximum number of trials $T_{\text {red }}$. In the following, simulation results will be presented and discussed for $T_{\text {red }} \in\{2,3,4\}$.

\section{Simulation Results}

In this section, we compare the performance of our proposed modified DSCF decoding algorithm against that of the original algorithm. The comparison is made in terms of average execution time, execution-time variance, and error-correction performance.

\section{A. Methodology}

The results are obtained using the same parameters as those described in Section IV except that the simulations are run for $10^{5}$ blocks or until the targeted minimum number of blocks in error is reached. The minimum number of errors is $10^{3}$ for an SNR range of $\{1.0,1.25, \ldots 2.25\} \mathrm{dB}$. Then the minimum number of errors is 500 and 300 for SNR points of $2.5 \mathrm{~dB}$ and $2.75 \mathrm{~dB}$ accordingly. Several reduced numbers of maximum trials $T_{\text {red }} \in\{2,3,4\}$ are simulated for the DSCF decoder 


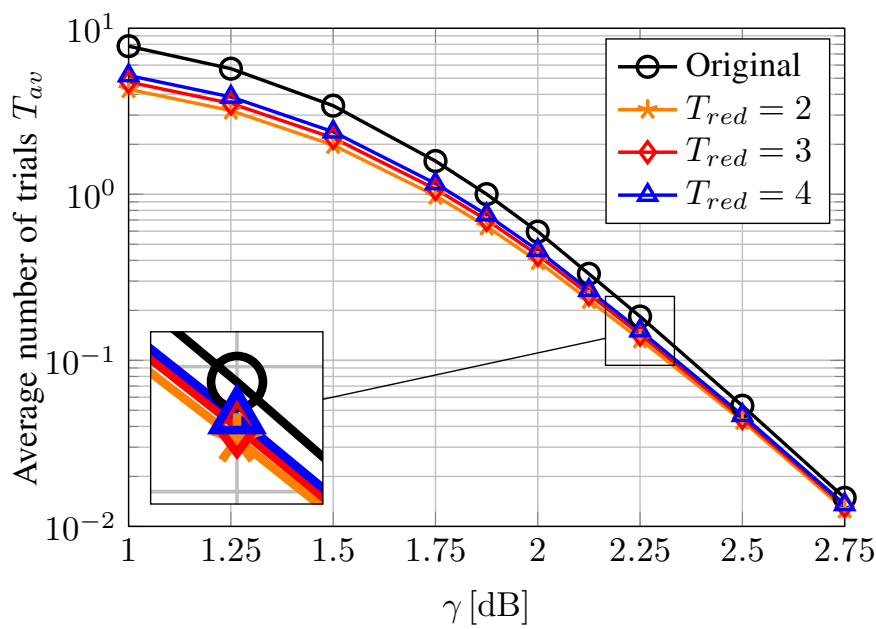

Fig. 3: Average number of trials, beyond the first SC decoding pass, for various SNR points for a DSCF decoder for $\mathcal{P}(1024,512)$ and $r=16$ with and without the proposed early-stopping mechanism.

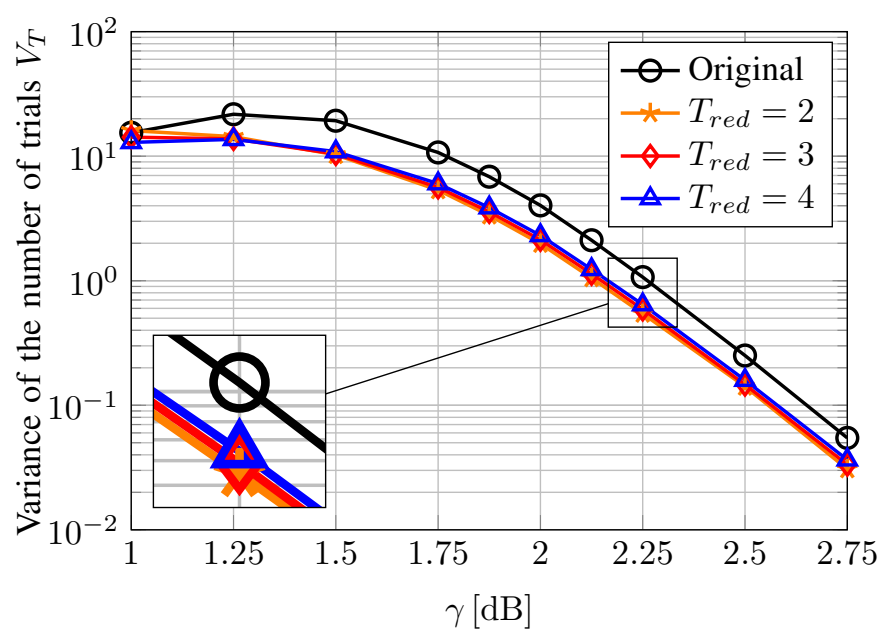

Fig. 4: Variance of the number of trials for various SNR points for a DSCF decoder for $\mathcal{P}(1024,512)$ and $r=16$ with and without the proposed early-stopping mechanism.

with the proposed early-stopping mechanism. The maximum number of trials beyond the initial SC decoding pass of the DSCF decoder is $T=10$. The value $c$ used in the calculation of the metrics associated to the bit-flipping candidates of Eq. [5] is 0.3 .

\section{B. Average Execution Time and Variance}

Fig. 3 shows the average execution time for the proposed DSCF decoder that incorporates our early-stopping mechanism, with $T_{\text {red }} \in\{2,3,4\}$. The average execution time for the original DSCF decoding algorithm is included for comparison. Similarly, Fig.4 shows the execution-time variance for our proposed decoding algorithm and includes that of the original DSCF algorithm for comparison. In both figures, the

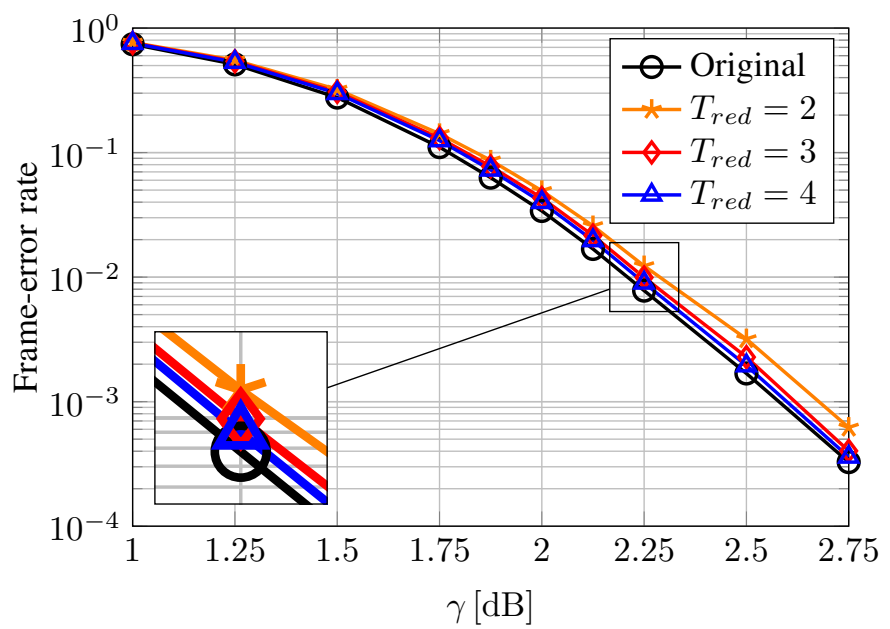

Fig. 5: FER of the DSCF decoder for $\mathcal{P}(1024,512)$ and $r=$ 16 with and without the proposed early-stopping mechanism.

original algorithm is depicted as a black curve with circle markers and the reminder of the curves are for our proposed modified algorithm.

From Fig.3, it can be seen that the proposed early-stopping mechanism leads to a reduction in the average number of trials compared to the original algorithm, regardless of the reduced maximum number of trials used. This reduction is more significant at low SNR and gradually vanishes as the channel condition improves. At the SNR $\gamma=2.25 \mathrm{~dB}$, which corresponds to a frame-error rate (FER) of $10^{-2}$ as will be shown in Fig [5, the reduction is of $22 \%$ for $T_{\text {red }}=3$. Another observation is that the differences between three curves corresponding to the modified DSCF are very small and the curve representing the decoder with $T_{\text {red }}=2$ achieves the smallest average execution time.

Looking at Fig.4, similarly to the average execution time, it can be seen that our proposed early-stopping mechanism leads to a reduction in the execution-time variance. Contrary to the reduction in the average execution time, the reduction in the execution-time variance is pretty similar across all SNR points. For the SNR point of interest, i.e., $\gamma=2.25 \mathrm{~dB}$, the reduction is of $45 \%$ for $T_{\text {red }}=3$. Again, we see that the differences between curves corresponding to $T_{\text {red }} \in\{2,3,4\}$ are very small.

\section{Error-Correction Performance}

Fig. 5 shows the error-correction performance in terms of FER of the modified DSCF decoder with the proposed earlystopping mechanism as well as that of the original DSCF decoder. Similarly to Figs. 3 and 4 the original DSCF algorithm is depicted as a black curve with circle markers and the reminder of the curves are for our modified algorithm. It can be seen that a reduced maximum number of trials $T_{\text {red }}$ of 2 with the codewords identified as likely undecodable leads to a coding loss that is little under $0.1 \mathrm{~dB}$ at a FER of $10^{-2}$ compared to the original DSCF algorithm. Increasing $T_{\text {red }}$ to 3 
significantly reduces that loss. The gap between the proposed algorithm and the original one is under $0.05 \mathrm{~dB}$ at the same FER. Increasing $T_{\text {red }}$ to 4 further reduces the error-correction loss but the improvement between $T_{\text {red }}=3$ and $T_{\text {red }}=4$ is not as important as the one created by going from 2 to 3 . For reference, we note that the FER of the DSCF decoder, with and without the proposed early-stopping algorithm, with $T=10$ falls between the FERs of a CRC-aided SCL decoder with list sizes $L$ of 2 and 4 .

Taking all three metrics into consideration-the average execution time, the execution-time variance, and the errorcorrection performance-, with a $\mathcal{P}(1024,512)$ polar code and a CRC of $r=16$ bits, using $T_{\text {red }}=3$ appears to offer the best tradeoff for a modified DSCF decoder that implements our proposed early-stopping mechanism.

\section{CONCLUSION}

In this work, we presented modifications to the original DSCF decoding algorithm to integrate an early-stopping mechanism that attempts to distinguish undecodable codewords from decodable. The key ingredients of this mechanism are the combination of an early-stopping metric with a precalculated threshold. Based on that metric, a codeword may be classified as likely undecodable, in which case the decoder attempts a reduced maximum number of trials, much lower than the initial maximal number of trials. After those trials, if decoding is not successful, the decoder stops. Compared to the original DSCF algorithm, in the region of interest for wireless communications, simulation results show that our proposed modifications could lead to reductions of $22 \%$ to the average execution time and of $45 \%$ to the execution-time variance at the cost of a minor error-correction loss of approximately $0.05 \mathrm{~dB}$. By reducing the execution-time variance, using this work contributes to keeping the amount of buffering required between the modules of a receiver tractable. Future work includes adapting the proposed early-stopping mechanism to the DSCF decoding algorithm with higher orders.

\section{ACKNOWLEDGEMENT}

The authors would like to thank Tannaz Kalatian for helpful discussions. This work was supported by an NSERC Discovery Grant (\#651824).

\section{REFERENCES}

[1] E. Arıkan, "Channel polarization: A method for constructing capacityachieving codes for symmetric binary-input memoryless channels," IEEE Trans. Inf. Theory, no. 7, pp. 3051-3073, Jul 2009.

[2] 3GPP, "NR; Multiplexing and channel coding," Tech. Rep. TS 38.212, Jan 2018, Release 15. [Online]. Available: http://www.3gpp.org/DynaReport/38-series.htm

[3] F. Ercan, C. Condo, S. Hashemi, and W. Gross, "On error-correction performance and implementation of polar code list decoders for 5G," in Ann. Allerton Conf. on Commun., Control, and Comput. (Allerton), Oct 2017, pp. 443-449.

[4] O. Afisiadis, A. Balatsoukas-Stimming, and A. Burg, "A low-complexity improved successive cancellation decoder for polar codes," in Asilomar Conf. on Signals, Syst. and Comput. (ACSSC), Nov 2014, pp. 2116-2120.

[5] P. Giard, A. Balatsoukas-Stimming, T. C. Müller, A. Bonetti, C. Thibeault W. J. Gross, P. Flatresse, and A. Burg, "PolarBEAR: A 28-nm FD-SOI ASIC for decoding of polar codes," IEEE J. Emerg. Sel. Topics Circuits Syst., vol. 7, no. 4, pp. 616-629, Dec. 2017.

[6] L. Chandesris, V. Savin, and D. Declercq, "Dynamic-SCFlip decoding of polar codes," IEEE Trans. Commun., no. 6, pp. 2333-2345, Jun 2018.

[7] F. Ercan, T. Tonnellier, N. Doan, and W. Gross, "Simplified dynamic SCFlip polar decoding," in IEEE Int. Conf. on Acoustics, Speech, and Signal Process. (ICASSP), May 2020.

[8] I. Tal and A. Vardy, "How to construct polar codes," IEEE Trans. Inf Theory, no. 10, pp. 6562-6582, Oct 2013. 